\title{
Heterogene digitale Kompetenzselbsteinschätzungen bei Lehramtsstudierenden
}

\section{Charlott Rubach und Rebecca Lazarides}

\section{Zusammenfassung}

Der vorliegende Beitrag fokussiert die digitalen Kompetenzselbsteinschätzungen Studierender in der Lehrkräftebildung. Hier wird der Frage nachgegangen, inwiefern es möglich ist, Lehramtsstudierende im Hinblick auf selbst eingeschätzte digitale Kompetenzen in Gruppen einzuteilen. Eine weitere Forschungsfrage ist, welche individuellen Merkmale sowie spezifischen Einstellungen und Nutzungsverhaltensweisen bezüglich digitaler Medien Studierende aufweisen. Daran anschließend werden Handlungsbedarfe für Strategien einer individuelle Lernvoraussetzungen aufgreifenden Lehrkräftebildung aus den Ergebnissen abgeleitet. Für die Analysen wurden Fragebogendaten von $\mathrm{N}=297$ Studierenden (61,3\% weiblich) deutscher Universitäten genutzt. Drei unterschiedliche Typen (Profile) Lehramtsstudierender können in Bezug auf digitale Kompetenzselbsteinschätzungen zum Umgang mit digitalen Medien und der Erschaffung digitaler Inhalte identifiziert werden. Die Profile unterscheiden sich signifikant in Bezug auf ihren Studienabschluss, ihre Einstellungen zur Nutzung digitaler Medien und ihr Nutzungsverhalten.

C. Rubach ( $\square)$

University of California Irvine, Irvine, CA, USA

E-Mail: crubach@uci.edu

R. Lazarides

Universität Potsdam, Potsdam, Deutschland

E-Mail: rebecca.lazarides@uni-potsdam.de 


\section{Schlüsselwörter}

Digitalisierung • Lehrkräftebildung • Werteüberzeugungen • TPCK • Will, Skill, Tool Model of Technology Integration

\section{$1 \quad$ Einleitung}

Im Rahmen der digitalen Transformation steht die Hochschulbildung vor der Herausforderung, digitale Kompetenzen bei Studierenden zu fördern, um diese bestmöglich auf ihr zukünftiges berufliches Umfeld vorzubereiten (Ehlers 2019; Graf-Schlattmann et al. 2019). Auch in der Lehrkräftebildung ist die Förderung digitaler Kompetenzen wichtig, da Lehramtsstudierende im Vergleich zu Studierenden anderer Fächer digitale Medien weniger nutzen und sich vergleichsweise als weniger kompetent im Umgang mit digitalen Medien einschätzen (Bertelsmann Stiftung 2017; Farjon et al. 2019). Eine zentrale Herausforderung für die Hochschulbildung ist vor diesem Hintergrund die Frage, wie digitale Kompetenzen und zugehörige Selbsteinschätzungen bei Lehramtsstudierenden im Rahmen der Lehrkräftebildung bestmöglich ausgebildet werden können.

Mit Fokus auf digitale Kompetenzen der Studierenden kann auf die Existenz verschiedener Kompetenzprofile geschlossen werden, beispielsweise Studierendengruppen mit geringen oder hohen Kompetenzen (Senkbeil et al. 2018). Die Identifikation solcher Gruppen steht bei Lehramtsstudierenden noch aus, gilt jedoch als notwendig für eine systematische und studierendenorientierte Förderung digitaler Kompetenzen und zugehöriger Kompetenzüberzeugungen von Lehramtsstudierenden. Der vorliegende Beitrag verfolgt vor diesem Hintergrund die Ziele, verschiedene Gruppen Lehramtsstudierender im Hinblick auf digitale Kompetenzselbsteinschätzungen zu identifizieren, zu beschreiben und im Anschluss Handlungsbedarf für eine individuelle Lernvoraussetzung aufgreifende Lehrkräftebildung abzuleiten. 


\section{Theoretische Grundlage}

\subsection{Interindividuell unterschiedliche Typen digitaler Kompetenzselbsteinschätzungen von Lehramtsstudierenden}

Der Begriff der digitalen Kompetenzen bezieht sich auf Fähigkeiten, Wissen und die Motivation, kompetent und mündig im digitalen Raum zu handeln - digitale Kompetenzen gelten in diesem Kontext als Teilbereich der Medienkompetenz (Eickelmann 2010). Digitale Kompetenzüberzeugungen können als Einschätzungen eigener Fähigkeiten zum kompetenten Handeln im digitalen Raum verstanden werden (Rubach und Lazarides 2019). Die Wahrnehmung eigener digitaler Kompetenzen ist für (angehende) Lehrkräfte hochrelevant, da diese einerseits eng mit den eigenen Kompetenzen zum Umgang mit digitalen Medien verbunden ist (Krumsvik 2014) und sich andererseits neben den Wertezuschreibungen auf das eigene Verhalten, beispielsweise auf den Einsatz digitaler Medien im Unterricht, auswirkt (Knezek und Christensen 2016).

In Anlehnung an die Strategie zur Bildung in der digitalen Welt (Kultusministerkonferenz 2016) können sieben Bereiche digitaler Kompetenzselbsteinschätzungen bei Lehramtsstudierenden identifiziert werden: Suchen und Verarbeiten; Kommunizieren und Kooperieren; Produzieren und Präsentieren; Schützen und sicher Agieren; Problemlösen und Handeln; Analysieren und Reflektieren sowie Unterrichten und Implementieren (Rubach et al. 2019). Die von der Kultusministerkonferenz erarbeitete Strategie (2016) wurde als Grundlage genutzt, um mithilfe einer einheitlichen Klassifizierung die Kompetenzselbsteinschätzungen der Lehramtsstudierenden bundesweit miteinander vergleichen zu können und eine systematische Identifizierung von Förderbedarf bei Studierenden zu ermöglichen.

Darüber hinaus verweisen empirische Ergebnisse auf unterschiedliche Kompetenzprofile. Kommer und Biermann (2012) konnten im Rahmen qualitativer Studien drei mediale Habitustypen von Lehramtsstudierenden unter Berücksichtigung der Kategorien Medienumgang, Werteüberzeugungen, Lernumwelten und Medienbesitz unterscheiden. Die Autoren beschreiben einen Typus Studierender, den sie als Gruppe der ambivalenten Bürgerlichen definieren - Personen in dieser Gruppe sind dem Einsatz neuer Medien gegenüber skeptisch eingestellt und technikdistanziert, was aus einer distanzierten Medienerziehung in der Familie resultiert. Der Typus der hedonistischen Pragmatiker*innen hingegen nutzt Technik, hat jedoch eine neutrale Einstellung zur Technik und ist geringfügig medienkritisch. Die Gruppe der kompetenten Medienaffinen bezieht 
sich auf Lehramtsstudierende (meist männlich) mit positiven Einstellungen zur Nutzung von Technik, sie setzen Technik vielfältig ein und verfügen über hohe Medienkompetenzen. Eine systematische quantitative Analyse von Typen digitaler Kompetenzselbsteinschätzungen bei Lehramtsstudierenden steht derzeit noch aus. Die Identifikation solcher Typen ist allerdings hochrelevant für die Lehrkräfteausbildung, da sich durch eine solche Typenbildung gruppenspezifische Bedürfnisse in der Hochschullehre identifizieren lassen. Das erlaubt, dazu passende Fördermaßnahmen im tertiären Bildungskontext zu entwickeln.

\subsection{Digitale Kompetenzselbsteinschätzungen, Nutzung digitaler Medien und Einstellungen zum Einsatz digitaler Medien}

Neben der Identifizierung von Kompetenzprofilen digitaler Kompetenzselbsteinschätzung ist die Validierung solcher Kompetenzprofile von Interesse. Eine Validierung bietet einerseits die Möglichkeit der empirischen Überprüfung identifizierter Typen und ermöglicht es andererseits, Typen durch Validierungsvariablen detaillierter zu beschreiben. Als Theoriegrundlage der hypothesengeleiteten Validierung der identifizierten Kompetenzprofile dienen das TPCK-Modell (Mishra und Koehler 2006) und das Will, Skill, Tool Model of Technology Integration (Knezek und Christensen 2016), da hier Kompetenzen und Kompetenzselbsteinschätzungen bezüglich der Nutzung digitaler Medien von (angehenden) Lehrkräften als Bedingungen für professionelles Unterrichtshandeln mit digitalen Medien beschrieben werden.

Das TPCK-Modell (Technological Pedagogical Content Knowledge) geht davon aus, dass das Zusammenspiel von technischem (TK), pädagogischem (PK) und inhaltlichem Wissen (CK) den effektiven Einsatz digitaler Medien im Unterricht erklärt. Angenommen wird, dass erst die Verknüpfung aus zwei und bestmöglich allen drei Wissensbereichen eine effektive und zielführende Integration von digitalen Medien in den Unterricht ermöglicht.

Ein zweites Modell zur Validierung der Kompetenzprofile ist das Will, Skill, Tool Model of Technology Integration von Christensen und Knezek (2016). Laut Modell steht die gelungene Integration digitaler Medien in den Unterricht in Zusammenhang mit dem Zugang, der Nutzung, den Kompetenzen sowie den Einstellungen der Lehrpersonen zum Einsatz digitaler Medien, beispielsweise dem Interesse oder den empfundenen Kosten (siehe auch Rubach et al. 2019; Drossel et al. 2018). 
Digitale Kompetenzselbsteinschätzungen stehen zudem in Zusammenhang mit individuellen Merkmalen von Studierenden. Verbunden mit geschlechtsspezifischen Sozialisationsprozessen scheinen Männer beispielsweise digitale Medien häufiger in den eigenen Unterricht zu integrieren und sich als digital kompetenter einzuschätzen als Frauen (Kommer und Biermann 2012; Drossel et al. 2018). Die Zusammenhänge zwischen Geschlecht, Nutzungsverhalten und digitalen Kompetenzselbsteinschätzungen lassen sich durch sozialisatorische Einflüsse erklären, zu denen beispielsweise die mediale Sozialisation in Schule und Privatleben gehört. Zudem wird ein Unterschied zwischen Bachelor- und Masterstudierenden bezüglich digitaler Kompetenzen und Kompetenzselbsteinschätzungen aufgrund zunehmender professionsbezogener Erfahrungen im Studienverlauf angenommen (Lemon und Garvis 2016; Şimşek und Sarsar 2019).

\subsection{Die vorliegende Studie}

Basierend auf bestehenden Forschungsarbeiten, die sich mit der Identifikation von Typen mit unterschiedlichen Kompetenzselbsteinschätzungen in Bezug auf den Umgang mit und der Nutzung von digitalen Medien im Hochschulkontext befassen (Kommer und Biermann 2012; Senkbeil et al. 2018), untersucht unsere Studie unterschiedliche Typen digitaler Kompetenzselbsteinschätzungen bei Lehramtsstudierenden. Es ergeben sich die folgenden Forschungsfragen:

1. Welche unterschiedlichen Typen lassen sich in Bezug auf digitale Kompetenzselbsteinschätzungen bei Lehramtsstudierenden identifizieren?

In Anlehnung an bestehende Forschungsbefunde (Kommer und Biermann 2012; Senkbeil et al. 2018) wird angenommen, dass interindividuell unterschiedliche Muster der digitalen Kompetenzselbsteinschätzungen bei Lehramtsstudierenden existieren, die über Niveauunterschiede zwischen den Teilbereichen der digitalen Kompetenzselbsteinschätzungen hinausgehen.

2. Unterscheiden sich die identifizierten Typen digitaler Kompetenzselbsteinschätzungen in Bezug auf das Geschlecht und den Studiengang der Studierenden?

Angenommen wird, dass männliche Lehramtsstudierende sowie Lehramtsstudierende im Masterstudium häufiger Typen mit hohen digitalen Kompetenzselbsteinschätzungen zugehörig sind im Vergleich zu weiblichen Lehramtsstudierenden und Lehramtsstudierenden im Bachelorstudium (Kommer und Biermann 2012; Lemon und Garvis 2016). 
3. Unterscheiden sich die identifizierten Typen digitaler Kompetenzselbsteinschätzungen in Bezug auf ihre Einstellungen zum Einsatz digitaler Medien im Unterrichtskontext sowie in Bezug auf die Nutzung digitaler Medien?

Erwartet wird, dass Profile mit höheren Kompetenzselbsteinschätzungen positivere Einstellungen zur Nutzung digitaler Medien im Unterricht haben und von umfangreicheren Vorerfahrungen in der Nutzung digitaler Medien berichten (Knezek und Christensen 2016).

\section{Methodisches Vorgehen}

\subsection{Stichprobe}

Für die Studie wurden Daten aus dem Projekt DigiKompEL 2017-2019 (Rubach und Lazarides 2019) genutzt. ${ }^{1}$

Die Datengrundlage der vorliegenden Studie umfasst $N=297$ Lehramtsstudierende der Sekundarstufe ${ }^{2}\left(61,3 \%\right.$ weiblich, Alter $\left.\bar{x}=25,28, S D^{3}=5,40\right)$, die zum Zeitpunkt der Befragung an deutschen Universitäten im Lehramtsstudium eingeschrieben waren. Die Daten stammen aus insgesamt sieben Bundesländern. ${ }^{4}$ Ein Großteil der Studierenden war im Bachelorstudium eingeschrieben $(60,6 \%)$. Am häufigsten wurden die Fächer Deutsch $(32,7 \%)$, Englisch $(23,6 \%)$ und Geschichte $(22,2 \%)$ studiert.

\subsection{Instrumente}

Selbst eingeschätzte digitale Kompetenzen. Die Skalen zur Erfassung von Fähigkeiten, die kompetentes Handeln von Lehramtsstudierenden im digitalen Raum abbilden, wurden von Rubach und Lazarides (2019) in Anlehnung an das Strategiepapier „Bildung in der digitalen Welt“ der Kultusministerkonferenz (2016)

\footnotetext{
${ }^{1}$ Detaillierte Informationen zur Stichprobe, Stichprobenziehung und Zielstellung des Projekts siehe Rubach und Lazarides (2020).

${ }^{2}$ Wir haben ausschließlich Lehramtsstudierende der Sekundarstufe genutzt, da diese Gruppe den Großteil der Gesamtstichprobe des DigiKompEL-Projekt ausmacht.

${ }^{3} \bar{x}=$ Mittelwert; SD $=$ Standardabweichung.

${ }^{4}$ Baden-Württemberg, Bayern, Berlin, Brandenburg, Mecklenburg-Vorpommern, Niedersachsen, Nordrhein-Westfalen.
} 
entwickelt. Alle Items ${ }^{5}$ wurden auf einer Likertskala von 1 [stimme gar nicht zu] bis 5 [stimme voll und ganz zu] eingeschätzt. Es wurden insgesamt sieben Kompetenzbereiche gemessen. Der erste Faktor Suchen und Verarbeiten wurde mit zwei Items erfasst $(\omega=, 68)$. Der zweite Faktor Kommunikation und Kooperieren wurde mit vier Items erfasst $(\omega=, 68)$. Zur Erfassung des dritten Faktors Produzieren und Präsentieren wurden zwei Items genutzt $(\omega=, 78)$. Der vierte Faktor, welcher inhaltlich den Bereich Schützen und sicher Agieren abbildet, wurde mit drei Items erfasst $(\omega=, 77)$. Um den fünften Faktor Problemlösen und Handeln zu erfassen, wurden vier Items genutzt $(\omega=, 89)$. Der sechste Faktor Analysieren wurde mit drei Items erfasst $(\omega=, 81)$. Zudem wurde ein siebter Faktor Unterrichten und Implementieren mit drei Items erfasst $(\omega=, 80)$.

Kompetenzselbsteinschätzung zum technisch-pädagogischen Inhaltswissen (TPCK, Technological Pedagogical Content Knowledge). Die Kompetenzselbsteinschätzung der Lehramtsstudierenden in Bezug auf die einzelnen Komponenten des TPCK-Modells wurden mittels vier Items ${ }^{5}$ von Chai, $\mathrm{Ng}, \mathrm{Li}$, Hong und Koh (2013) mit einer fünfstufigen Antwortskala von 1 [stimme gar nicht zu] bis 5 [stimme voll und ganz zu] erfasst. Es wurde das technische Fachwissen (TCK), das technisch-pädagogische Wissen (TPK) sowie das technische, pädagogische und inhaltliche Fachwissen (TPCK) abgebildet.

Werteüberzeugungen bei der Nutzung digitaler Medien im Unterricht. Entsprechend der Ausführungen von Eccles (2005) wurden auf der eigens entwickelten Skala die Werteüberzeugungen, also die empfundene Nützlichkeit, Wichtigkeit, das Interesse und die Kosten für die Nutzung digitaler Medien im eigenen Unterricht, erfasst (Rubach und Lazarides 2019). Die Erfassung aller Teilbereiche erfolgte durch jeweils drei Items ${ }^{5}$ mit einer fünfstufigen Antwortskala von 1 [stimme gar nicht zu] bis 5 [stimme voll und ganz zu]. Für die Analyse wurde ein Faktor bestehend aus den drei Teilbereichen zum Interesse, zur Nützlichkeit und Wichtigkeit gebildet, welcher die durchschnittlich empfundenen Werteüberzeugungen zum Einsatz digitaler Medien abbildet. Die Reliabilität dieses Faktors war mit $\omega=, 91$ gut. Die empfundenen Kosten für die Nutzung digitaler Medien im Unterricht werden in einer weiteren Skala als Komponente relevanter Werteüberzeugungen erfasst. Die Reliabilität dieser Skala war mit $\omega=$ ,69 zufriedenstellend.

Einstellungen zur Nutzung digitaler Medien. Die Erfassung positiver und negativer Einstellungen zur Nutzung digitaler Medien als Lehrkraft wurden in

\footnotetext{
${ }^{5}$ Zusätzliche Informationen zu genutzten Items (Anhang A1) sowie den Kompetenzbereichen und den zugehörigen Kompetenzstufen (Anhang A2) können unter https://osf.io/xzyeu/ (https://doi.org/10.17605/OSF.IO/XZYEU) abgerufen werden.
} 
Anlehnung an Davis (1989) entwickelt. Genauer wurde die Vielfalt der von Davis (ebenda) beschriebenen jobspezifischen Einstellungen aufgegriffen und auf den aktuellen Themenbereich der Nutzung digitaler Medien im Unterricht übertragen. Es wurden vier Items ${ }^{5}$ genutzt, welche ein fünfstufiges Antwortformat von 1 [stimme gar nicht $\mathrm{zu}$ ] bis 5 [stimme voll und ganz zu] hatten. Es wurden die empfundene Überforderung durch und die Vertrautheit mit dem Einsatz digitaler Medien im Unterricht sowie die Überschätzung der Relevanz des Einsatzes digitaler Medien im Unterricht und die empfundene Relevanz der Auseinandersetzung mit digitalen Medien für Lehrkräfte erfasst.

Nutzung digitaler Medien. Die Erfassung der vielfältigen Nutzung unterschiedlicher digitaler Medien erfolgt durch eine dichotomisierte Abfrage (ja/nein) - hier wurden vier Endgeräte (zum Beispiel Computer, Tablet) sowie 14 Tools beziehungsweise Kategorien von Tools (zum Beispiel Clouds, Weblogs) aufgelistet, wobei angegeben werden sollte, inwieweit diese regelmäßig privat oder im Studium genutzt werden. Darüber hinaus gab es in einem offenen Antwortformat die Möglichkeit, Geräte und Tools einzutragen, welche nicht vorgegeben waren. Zur weiteren Analyse wurden Summenwerte gebildet, welche die Vielfältigkeit der Nutzung digitaler Medien darstellen.

\subsection{Methodisches Vorgehen}

Zur statistischen Analyse wurden die Programme SPSS 26 sowie Mplus 8.1 (Muthén und Muthén 1998-2016) genutzt. Zur Identifikation unterschiedlicher Typen wurden latente Profilanalysen berechnet, welche ermöglichen, einzelne Gruppen in Bezug auf die digitalen Kompetenzselbsteinschätzungen zu identifizieren. Das schrittweise Vorgehen richtete sich an die von Morin et al. (2016) beschriebene Factor Mixture Analysis (FMA). Die Schritte der Datenanalyse werden im Anhang $\mathrm{A} 3^{6}$ detailliert beschrieben.

\footnotetext{
${ }^{6}$ Zusätzliche Methoden- und Ergebnisbeschreibungen (Anhang A3) können unter https://osf. io/xzyeu/ (https://doi.org/10.17605/OSF.IO/XZYEU) abgerufen werden.
} 


\section{$4 \quad$ Ergebnisse}

\subsection{Ergebnisse der latenten Profilanalysen}

Zur Beantwortung der ersten Fragestellung wurde eine Serie von Factor-MixtureProfilanalysen mit Modelllösungen für bis zu acht Klassen berechnet. Die Modellgütekriterien der acht Klassenlösungen sowie die Interpretation der Lösung sind im Anhang $\mathrm{A} 3^{6}$ dargestellt.

Im Folgenden werden die einzelnen Profile im Detail beschrieben: Profil A (12,1 \% der Lehramtsstudierenden) wird als Profil mit moderaten professionsbezogenen Basiskompetenzen und geringen Fähigkeiten zur Gestaltung digitaler Inhalte benannt. Studierende dieses Profils zeichnen sich vor allem dadurch aus, dass sie im Vergleich zu den Studierenden anderer Profile signifikant geringere Einschätzungen in den Kompetenzbereichen aufweisen, welche das Gestalten und Entwickeln digitaler Inhalte abbilden (Kompetenzbereiche: Problemlösen und Handeln, Produzieren und Präsentieren). Zudem schätzen sich Lehramtsstudierende dieses Profils als weniger kompetent im Vergleich zu Studierenden anderer Profile darin ein, digitale Medien und Inhalte in Bezug auf Nutzungsmöglichkeiten, Chancen und Risiken zu analysieren und kritisch zu reflektieren sowie im digitalen Raum zu kommunizieren und digitale Inhalte zu verbreiten (Kompetenzbereiche: Analysieren und Reflektieren, Kommunizieren und Kooperieren).

Dem Profil B (33,7 \% der Lehramtsstudierenden) sind Studierende zugeordnet, die ähnlich wie die Studierenden in Profil A moderate professionsbezogene Basiskompetenzen angeben. Ihre Fähigkeitseinschätzungen in Bezug auf das Erkennen, Klassifizieren, Ausführen und Bewerten von Quellen digitaler Informationen, digitaler Inhalte, von Schutzmaßnahmen ihrer Privatsphäre und Gesundheit im digitalen Raum sowie von lern- und lehrbezogenen Potenzialen digitaler Medien für den eigenen Fachunterricht sind moderat ausgeprägt (Kompetenzbereiche: Suchen und Verarbeiten, Schützen und sicher Agieren, Unterrichten und Implementieren). Im Vergleich zu Studierenden des Profils A haben Studierende des Profils B höhere Einschätzungen in den Kompetenzbereichen Problemlösen und Handeln, Produzieren und Präsentieren, Analysieren und Reflektieren sowie Kommunizieren und Kooperieren. Diese Kompetenzeinschätzungen sind jedoch geringer im Vergleich zu Studierenden des Profils C. Daher wird das Profil B als Profil mit moderaten professionsbezogenen Basiskompetenzen und moderaten Fähigkeiten zur Gestaltung digitaler Inhalte bezeichnet.

Dem Profil C (54,2 \% der Lehramtsstudierenden) sind Studierende zugeordnet, die in allen sieben Kompetenzbereichen im Vergleich zu Studierenden der Profile 
A und B höhere Fähigkeitseinschätzungen aufweisen. Daher wird das Profil als Profil mit hohen professionsbezogenen Basiskompetenzen und hohen Fähigkeiten zur Gestaltung digitaler Inhalte benannt.

In allen sieben Kompetenzselbsteinschätzungsskalen zeigen sich signifikante Unterschiede zwischen den drei Profillösungen (siehe Tab. 1). Mit Fokus auf Niveauunterschiede zwischen den Kompetenzselbsteinschätzungen zeigt sich, dass sich die Studierenden in den Bereichen Suchen und Verarbeiten, Koтmunizieren und Kooperieren, Schützen und sicher Agieren und Unterrichten und Implementieren über alle drei Profile hinweg durchschnittlich am kompetentesten einschätzen, da die Mittelwerte in allen drei Profilen über oder nah am Wert von $\bar{x}=3,5$ liegen. Interessant sind Mittelwertdifferenzen zwischen den Profillösungen. Die geringsten Mittelwertdifferenzen zwischen den Profilen zeigen sich in den Bereichen Suchen und Verarbeiten $(\Delta \bar{x}=0,56)$, Schützen und sicher Agieren $(\Delta \bar{x}=0,62)$ sowie Unterrichten und Implementieren $(\Delta \bar{x}=0,66)$. Die größten Mittelwertdifferenzen zwischen den Profilen zeigen sich in den Kompetenzbereichen Produzieren und Präsentieren $(\Delta \bar{x}=2,12)$ und Problemlösen und Handeln $(\Delta \bar{x}=1,33)($ Abb. 1$)$.

\subsection{Validierung der Profile}

Zur Validierung der Profile wurden im ersten Schritt die TPCKSelbsteinschätzungen genutzt. Hier wurden zwei Niveaustufen von professionsbezogenen Fähigkeiten zum Einsatz digitaler Medien im Unterricht abgefragt: Die geringere Niveaustufe erfasst die Kombination aus zwei Wissensbereichen, genauer das technische Wissen mit a) dem pädagogischen Wissen (TPK) oder b) mit dem Fachwissen (TCK). Die höchste Kompetenzstufe ist die Kombination aller drei Wissensbereiche, genauer der Einsatz digitaler Medien unter Nutzung des pädagogischen Wissens und Fachwissens (TPCK). Die Ergebnisse sind in Tab. 2 dargestellt. Wie erwartet berichten Studierende im Profil $\mathrm{C}$ die höchsten Kompetenzselbsteinschätzungen auf allen Niveaustufen des TPCK-Modells im Vergleich zu Studierenden in Profil A und B. Interessant ist, dass sich Studierende der Profile A und B nicht in den unteren Kompetenzstufen unterscheiden (TPK, TCK), jedoch Studierende des Profils A signifikant geringere Einschätzungen in den Items der höheren Kompetenzstufe (TPCK) aufweisen als Studierende im Profil B. Das weist darauf hin, dass Studierende im Profil A sich weniger fähig fühlen, alle drei Wissensbereiche zu verbinden und digitale Medien zur Nutzung ihres pädagogischen Wissens und fachlichen Wissens einzusetzen. 


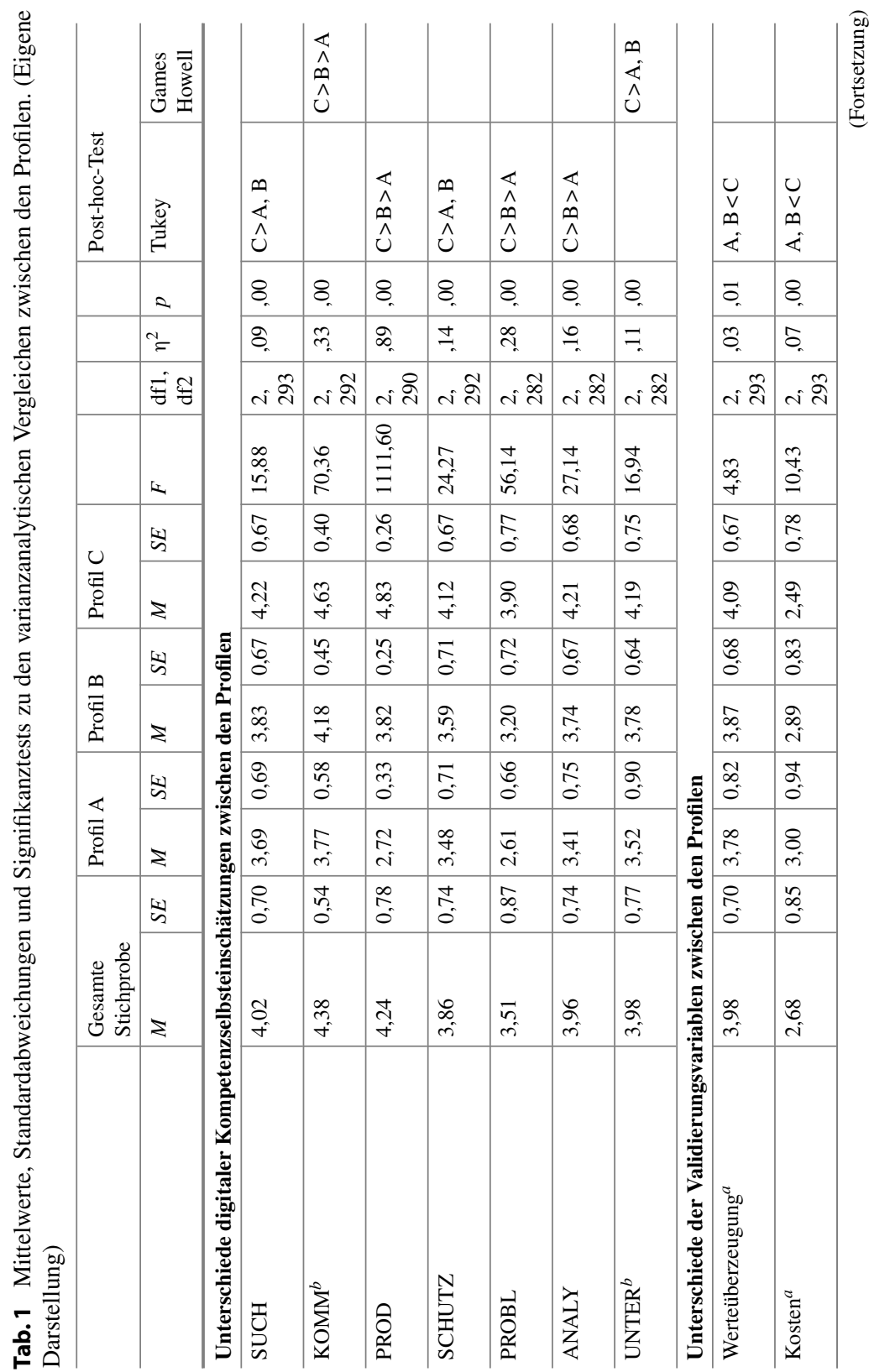




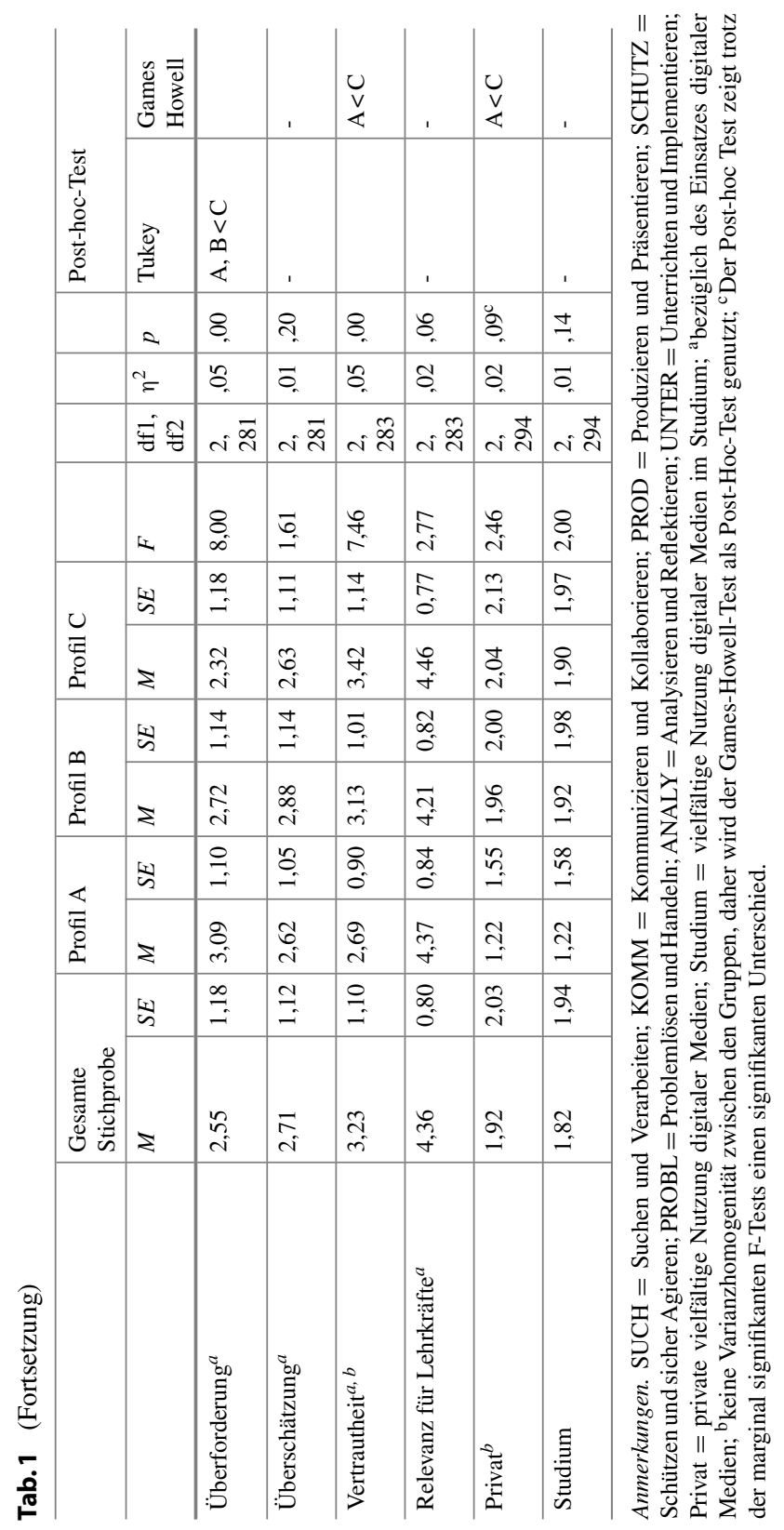




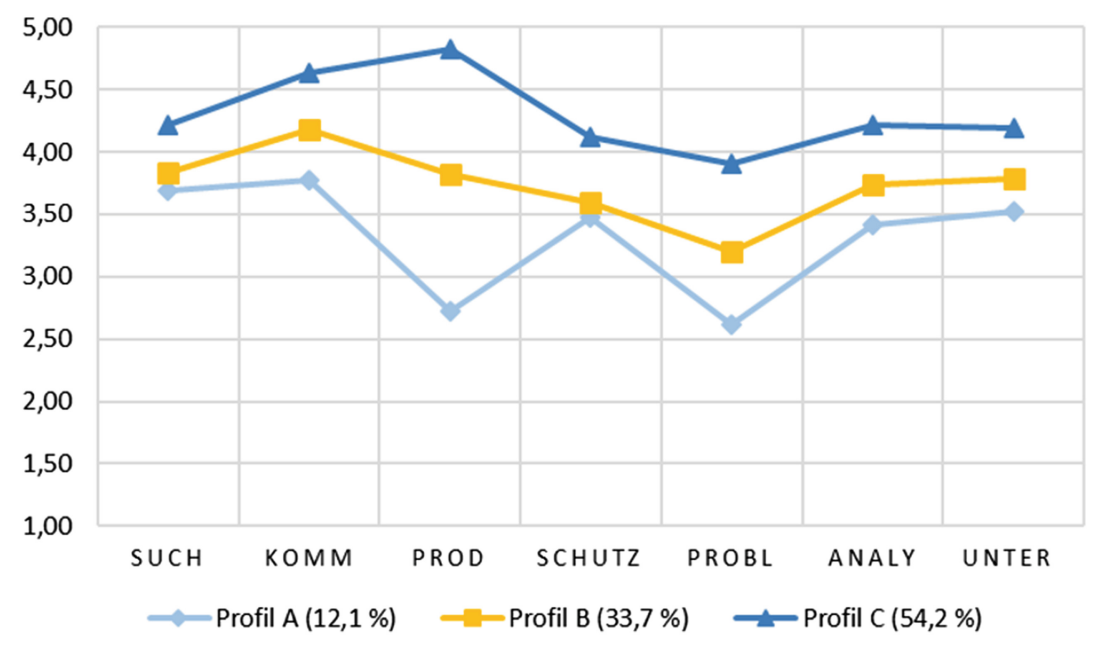

Abb. 1 Darstellung der drei latenten Profile mit den geschätzten Mittelwerten in den sieben Skalen der digitalen Kompetenzselbsteinschätzungen. (Eigene Darstellung)

Zur detaillierteren Definition der einzelnen Profile wurden weitere Unterschiedsanalysen zwischen den Profilen mit theoretisch beschriebenen Validierungsvariablen vorgenommen. Es zeigen sich keine signifikanten Unterschiede in der Verteilung von Geschlechtern zwischen den drei Profilen $\left(\chi^{2}(2)=0,75\right.$, $p>, 05)$. Jedoch sind in den Profilen mit den höheren digitalen Kompetenzselbsteinschätzungen (Profil B, Profil C) im Vergleich zum Profil A proportional mehr Bachelorstudierende als Masterstudierende zugehörig $\left(\chi^{2}(2)=9,00, p<, 05\right)$.

Analysen zu den Einstellungen und zum Nutzungsverhalten der Typen zeigen (siehe Tab. 1), dass Studierende in Profil A und B den Einsatz digitaler Medien im Unterricht als weniger interessant, nützlich und wichtig im Vergleich zu Studierenden des Profils C empfinden. Studierende in den Profilen A und B empfinden signifikant höhere psychologische Kosten bei der Nutzung digitaler Medien im Vergleich zu Studierenden im hochkompetenten Profil C. Das bedeutet, dass Studierende in den Profilen A und B mit dem Einsatz digitaler Medien mehr Zeitaufwand und Bedenken assoziieren und sich als überforderter einschätzen, digitale Medien im Unterricht einzusetzen. Interessant ist, dass die Studierenden aller Profile der Auseinandersetzung mit digitalen Medien für die spätere Rolle als Lehrkräfte den gleichen Wert beimessen und die Relevanz der Auseinandersetzung als wenig überschätzt wahrnehmen. 


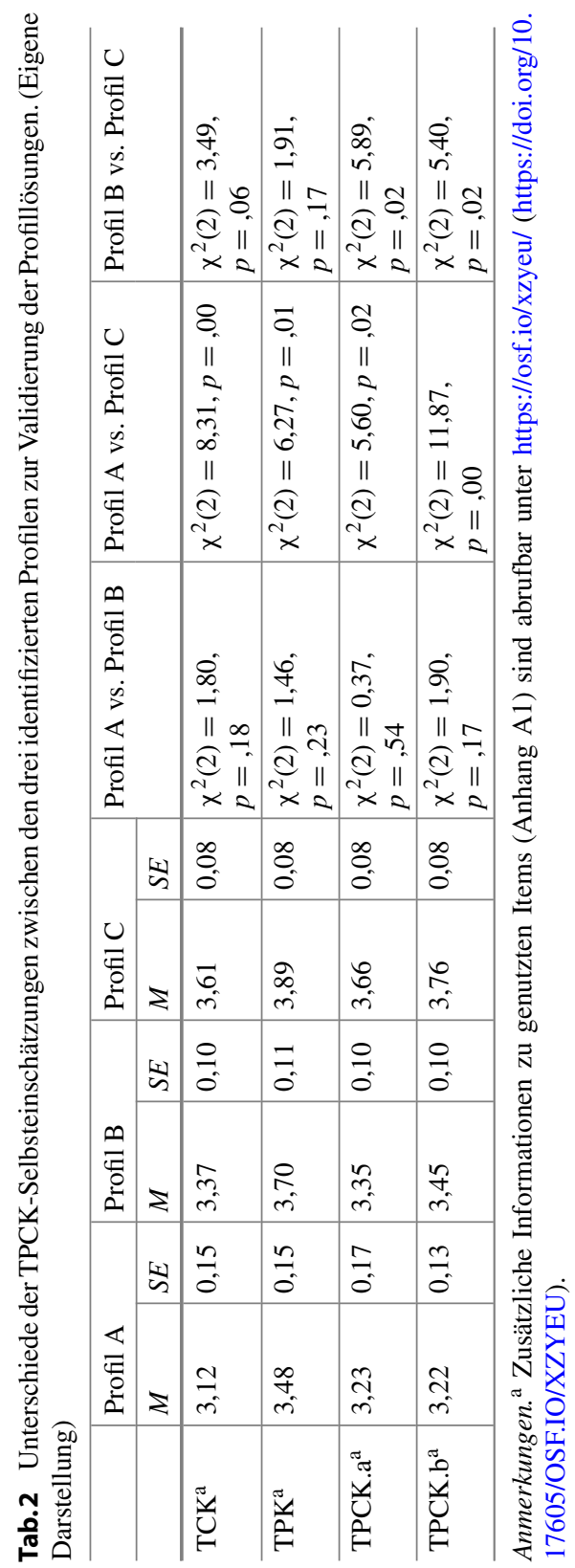


In Bezug auf Vorerfahrungen in der Nutzung von digitalen Medien zeigt sich, dass es einen Anstieg der berichteten Vertrautheit mit dem Einsatz digitaler Medien im Unterricht von Profil A über Profil B zu Profil C gibt. Studierende im hochkompetenten Profil $\mathrm{C}$ sind beispielsweise vertrauter im Umgang mit digitalen Medien im Vergleich zu Profil A und Profil B. Diese Tendenz zeigt sich auch bei der vielfältigen Nutzung von digitalen Medien im privaten Umfeld und im Studium, sodass Studierende aus Profil A beispielsweise digitale Medien weniger vielfältig im privaten Umfeld nutzen im Vergleich zu Studierenden im Profil C.

\section{Diskussion}

In der vorliegenden Studie wurde überprüft, welche unterschiedlichen Typen bei Lehramtsstudierenden in Bezug auf ihre digitalen Kompetenzselbsteinschätzungen identifiziert werden können. Zusammenfassend können drei unterschiedliche Typen bestimmt werden: Ungefähr die Hälfte der Lehramtsstudierenden lässt sich dem Profil mit hohen professionsbezogenen Basiskompetenzen und hohen Fähigkeiten zur Gestaltung digitaler Inhalte (Profil C) zuordnen. Diese Studierenden schätzen ihre eigene Kompetenz in allen sieben Kompetenzbereichen höher ein im Vergleich zu Studierenden in Profil A und B. Weiterhin zeigte sich, dass ungefähr ein Drittel der Studierenden sich einem Profil mit moderaten professionsbezogenen Basiskompetenzen und moderaten Fähigkeiten zur Gestaltung digitaler Inhalte (Profil B) zuweisen lassen und etwas mehr als ein Zehntel der Befragten ein Profil mit moderaten professionsbezogenen Basiskompetenzen und geringen Fähigkeiten zur Gestaltung digitaler Inhalte (Profil A) aufweisen. Studierende, welche dem Profil A zugeordnet sind, berichten im Vergleich zu Studierenden im Profil B und $\mathrm{C}$ von signifikant geringeren Kompetenzselbsteinschätzungen in allen sieben Kompetenzbereichen.

Die Unterscheidung dreier Gruppen Studierender im Hinblick auf ihre Medienkompetenzen wurde bei Senkbeil et al. (2018) sowie Kommer und Biermann (2012) deutlich. Unsere Ergebnisse schließen an die empirischen Vorarbeiten an und erweitern bisherige Arbeiten dahin gehend, dass Lehramtsstudierende auch aufgrund ihrer digitalen Kompetenzselbsteinschätzungen den drei unterschiedlichen Kompetenzprofilen zugeordnet werden können. Die Relevanz der Identifikation solcher Typen zeigt sich darin, dass konkrete Bedürfnisse und damit 
verbundene Schwächen und Stärken von Studierenden in den einzelnen Gruppen deutlich werden, die in der Lehrkräftebildung adressiert werden sollten. ${ }^{7}$

\subsection{Praktische Implikationen für die inhaltliche Gestaltung der Hochschul- und Lehrkräftebildung}

Zusammenfassend ergeben sich folgende Implikationen für die inhaltliche Gestaltung der Lehrkräftebildung aus den Ergebnissen unserer Studie:

1. Sichergestellt werden sollte, dass angehende Lehrkräfte sich kompetent in den sieben identifizierten Themenfeldern einschätzen (KMK 2016; Rubach und Lazarides 2019). Die Relevanz solcher Kompetenzselbsteinschätzungen zum Umgang mit digitalen Medien zeigt sich insbesondere darin, dass diese mit den eigenen Kompetenzen im Umgang mit digitalen Medien assoziiert sind und als Grundlage der digital gestützten Unterrichtsgestaltung gelten (Knezek und Christensen 2016; Krumsvik 2014). Insbesondere die Themenfelder des Problemlösens und Handelns, Produzierens und Präsentierens sowie Schützens und sicher Agierens bedürfen eventuell besonderer Aufmerksamkeit, da hier in unseren Daten die geringsten Kompetenzselbsteinschätzungen für Studierende beziehungsweise die größten Unterschiede zwischen Studierenden bestehen. Daher wird vorgeschlagen, Lehrpläne dahin gehend anzupassen und die Förderung der Kompetenzselbsteinschätzungen in den sieben Kompetenzbereichen als Kompetenz- und Lernziele im Curriculum der Lehrkräftebildung zu verankern (van Ackeren et al. 2019).

2. Zwischen den drei Profilen zeigen sich signifikant unterschiedliche Werteüberzeugungen hinsichtlich des Einsatzes digitaler Medien im Unterricht. Interessant ist dabei, dass hohe Kompetenzselbsteinschätzungen mit höherem Interesse und Nutzenüberzeugungen einhergehen, während geringe und moderate Kompetenzselbsteinschätzungen zum Einsatz von Medien im Unterricht eher mit der Befürchtung von mehr Zeitaufwand und Überforderung assoziiert

\footnotetext{
${ }^{7}$ Statistisch wird insbesondere im Vergleich der Gesamtmittelwerte und der Mittelwerte der einzelnen Skalen zwischen den Profilen die Relevanz der Identifikation einzelner Profile deutlich. Aufgrund der Gesamtmittelwerte würde man davon ausgehen, dass Studierende sich im Kompetenzbereich Produzieren und Präsentieren mit einem Mittelwert von $\bar{x}=4.24$ $(\mathrm{SD}=0.78)$ als kompetent einschätzen. Durch die Profilanalyse wird jedoch deutlich, dass hier die größten Differenzen in der Einschätzung der Studierenden bestehen, sodass sich die Hälfte der Studierenden durchaus kompetent wahrnimmt, die andere Hälfte in diesem Bereich jedoch Förderbedarf aufweist (siehe Tab. 1).
} 
sind. Für die Lehrkräftebildung resultiert daraus die Notwendigkeit, Studierenden den Wert des Medieneinsatzes aufzuzeigen. Studien zeigen effektive Möglichkeiten der Veränderung von Einstellungen zu digitalen Medien bei Lehrkräften auf (Levin und Wadmany 2006). Ein Ansatz für die Lehrkräftebildung ist beispielsweise, mit Lehramtsstudierenden den Mehrwert digitaler Medien für eigene Unterrichtsprozesse sowie für die Kompetenzförderung zukünftiger Schüler*innen auf Grundlage empirischer Evidenzen zu diskutieren und zu reflektieren (Ottenbreit-Leftwich et al. 2010). Beispiele dafür sind videobasierte Reflexionsverfahren (siehe dazu Kießler 2020) sowie auf E-Portfolios und der Nutzung von Peer-Assessments basierende Reflexionsprozesse (Berg 2018). Weiterhin können Einstellungen zur aktiven Auseinandersetzung mit digitalen Medien verändert werden, indem Lernumwelten den zu fördernden Einstellungen entsprechend gestaltet werden (zum Beispiel technologiereiche Lernumgebungen) und Lehramtsstudierende in unterschiedlichen Rollen digitale Medien nutzen, den Mehrwert des Einsatzes digitaler Medien reflexiv erkennen und sich aktiv mit den zu befürchtenden Kosten auseinandersetzen können (Rubach et al. 2019; Ertmer et al. 2012; Levin und Wadmany 2006).

\subsection{Praktische Implikationen für die pädagogische Gestaltung der Hochschul- und Lehrkräftebildung}

Mit dem Ziel, dass Lehramtsstudierende sich digital kompetent einschätzen, sollte die universitäre Lehrkräftebildung die aufgezeigte interindividuelle Heterogenität in den digitalen Kompetenzselbsteinschätzungen der Lehramtsstudierenden aufgreifen.

1. Studierende müssen im Rahmen der Ausbildung digitale Medien nutzen und eigene Erfahrungen mit Nutzen und Kosten für spätere Unterrichtsprozesse reflektieren (Rubach et al. 2019; Levin und Wadmany 2006).

2. Besonders relevant ist die Überlegung, wie Studierende des Profils mit moderaten professionsbezogenen Basiskompetenzen und moderaten Fähigkeiten zur Gestaltung digitaler Inhalte sowie des Profils mit moderaten professionsbezogenen Basiskompetenzen und geringen Fähigkeiten zur Erschaffung digitaler Inhalte bestmöglich gefördert werden können. Eine Möglichkeit ist das Prinzip des Lernens am Modell: Dozierende nutzen digitale Medien in der Lehre, sodass Studierende die Möglichkeit erhalten, Einsatzmöglichkeiten verschiedener Medien kennenzulernen. Wichtig dabei ist, dass Studierende digitale 
Medien aktiv nutzen und diese Erfahrungen für spätere Unterrichtsprozesse reflektieren (Rubach et al. 2019; Ertmer et al. 2012).

3. Für alle Lehramtsstudierenden, insbesondere für die Studierenden mit höherer Kompetenzselbsteinschätzung, sollten Lehrformate mit einem handlungsorientierten Prinzip implementiert werden: Studierende werden als Medienproduzierende gesehen (Hug 2011) und sollten einerseits die Möglichkeit der selbstständigen Nutzung digitaler Medien erhalten und andererseits den Einsatz digitaler Medien für eigene Unterrichtsprozesse planen, praktisch üben und reflektieren (Rubach et al. 2019; Bastian und Aufenanger 2015). Van Ackeren et al. (2019) sprechen hier in Anlehnung an das TPCK-Modell (Mishra und Koehler 2006) von der Notwendigkeit einer verzahnten Kompetenzentwicklung, sodass Bildungs- und Fachwissenschaften gemeinsam im Zuge der Verbindung von pädagogischem, inhaltlichem und technischem Wissen eine bestmögliche Vorbereitung von Studierenden gewährleisten (siehe auch Rubach et al. 2019).

\subsection{Limitationen und Ausblick}

Die Ergebnisse der vorliegenden Studie können nur unter Berücksichtigung ihrer Limitationen interpretiert werden. Die Teilnahme der Studierenden an der Befragung war freiwillig. So könnte es sein, dass Studierende teilnahmen, die schon ein hohes Interesse am Thema Digitalisierung haben und ihre Fähigkeiten eventuell eher gut einschätzen. Eine weitere Limitation ist, dass in der vorliegenden Stichprobe nur Lehramtsstudierende der Sekundarstufe vertreten sind. Daher wird ausdrücklich empfohlen, eine Replikationsstudie durchzuführen, welche Studierende verschiedener Schulstufen gleichermaßen in einer umfassenden Stichprobe berücksichtigt. So können Befunde dieser Studie mit Studierenden aller Kompetenz- und Motivationsniveaus verifiziert werden. Auch der Einsatz standardisierter Tests zur Messung der digitalen Kompetenzen von Lehramtsstudierenden wäre wünschenswert, um die Selbsteinschätzungen der Profile mit Ergebnissen von Kompetenztests vergleichen zu können (Brandhofer 2017). Zudem möchten wir vorschlagen, dass zukünftige Forschungsprojekte eine stringentere Messung der Nutzung digitaler Tools vornehmen, sodass entweder konkrete Tools (zum Beispiel Moodle) oder Kategorien von Tools (zum Beispiel Lernmanagementsysteme) erfasst werden. 


\section{Literatur}

Bastian, J., \& Aufenanger, S. (2015). Medienbezogene Vorstellungen von (angehenden) Lehrpersonen. In M. Schiefner-Rohs, C. Gómez Tutor, \& C. Menzer (Hrsg.), Lehrer.Bildung.Medien. Herausforderungen für die Entwicklung und Gestaltung von Schule. Grundlagen der Berufs- und Erwachsenenbildung, 82 (S. 19-34). Baltmannsweiler: Schneider-Verlag Hohengehren.

Berg, G. (2018). Die Digitalisierung universitären Lehr-Lernens in der Lehrkräftebildung. Das Projekt [D-3] an der Martin-Luther-Universität Halle-Wittenberg. In B. Getto, P. Hintze, \& M. Kerres (Hrsg.), Digitalisierung und Hochschulentwicklung. Proceedings zur 26. Tagung der Gesellschaft für Medien in der Wissenschaft e. V. Medien in der Wissenschaft, 74 (S. 213-221). Münster: Waxmann.

Bertelsmann Stiftung. (2017). Studierende sind keine digitalen Enthusiasten. https://www. bertelsmann-stiftung.de/de/presse/pressemitteilungen/pressemitteilung/pid/studierendesind-keine-digitalen-enthusiasten/. Zugegriffen: 16. März 2017.

Brandhofer, G. (2017). Lehr-/Lerntheorien und mediendidaktisches Handeln. Eine Studie zu den digitalen Kompetenzen von Lehrenden an Schulen. Wissenschaftliche Beiträge aus dem Tectum-Verlag, 42, (1. Aufl.). Marburg: Tectum.

Chai, C. S., Ng, E. M. W., Li, W., Hong, H.-Y., \& Koh, J. H. L. (2013). Validating and modelling technological pedagogical content knowledge framework among asian preservice teachers. Australasian Journal of Educational Technology, 29(1), 41-53. https://doi. org/10.14742/ajet.174.

Davis, F. D. (1989). Perceived usefulness, perceived ease of use, and user acceptance of information technology. MIS Quarterly, 13(3), 319. https://doi.org/10.2307/249008.

Drossel, K., Eickelmann, B., Schaumburg, H. \& Labusch, A. (2019). Nutzung digitaler Medien und Prädiktoren aus der Perspektive der Lehrerinnen und Lehrer im internationalen Vergleich. In B. Eickelmann, W. Bos, J. Gerick, F. Goldhammer, H. Schaumburg, M. Senkbeil et al. (Hrsg.), ICILS 2018 \#Deutschland. Computer- und informationsbezogene Kompetenzen von Schülerinnen und Schülern im zweiten internationalen Vergleich und Kompetenzen im Bereich Computational Thinking (S. 205-240). Münster: Waxmann.

Eccles, J. S. (2005). Subjective task value and the Eccles et al. model of achievement-related choices. In A. J. Elliot \& C. S. Dweck (Hrsg.), Handbook of competence and motivation (Paperback ed.) (S. 105-121). New York: Guilford Publications.

Eccles, J. S., Adler, T. F., Futterman, R., Goff, S. B., Kaczala, C. M., Meece, J. L., \& Midgley, C. (1983). Expectancies, values, and academic behaviors. In J. T. Spence (Hrsg.), Achievement and achievement motivation (S. 75-146). San Francisco: W. H. Freeman.

Ehlers, U.-D. (2019). Future Skills und Hochschulbildung „Future Skill Readiness“. In J. Hafer, M. Mauch, \& M. Schumann (Hrsg.), Teilhabe in der digitalen Bildungswelt. Medien in der Wissenschaft, 75, (1. Aufl.) (S. 37-48). Münster: Waxmann.

Eickelmann, B. (2010). Digitale Medien in Schule und Unterricht erfolgreich implementieren. Eine empirische Analyse aus Sicht der Schulentwicklungsforschung. Empirische Erziehungswissenschaft, 19. (Dissertation, Technische Universität Dortmund). Münster: Waxmann. 
Ertmer, P. A., Ottenbreit-Leftwich, A. T., Sadik, O., Sendurur, E., \& Sendurur, P. (2012). Teacher beliefs and technology integration practices: A critical relationship. Computers \& Education, 59(2), 423-435. https://doi.org/10.1016/j.compedu.2012.02.001.

Farjon, D., Smits, A., \& Voogt, J. (2019). Technology integration of pre-service teachers explained by attitudes and beliefs, competency, access, and experience. Computers \& Education, 130, 81-93. https://doi.org/10.1016/j.compedu.2018.11.010.

Graf-Schlattmann, M., Meister, D. M., Oevel, G., \& Wilde, M. (2019). Digitalisierungsstrategien auf dem Prüfstand. Eine empirische Untersuchung auf Basis der Grounded-TheoryMethodologie an deutschen Hochschulen. In J. Hafer, M. Mauch, \& M. Schumann (Hrsg.), Teilhabe in der digitalen Bildungswelt. Medien in der Wissenschaft, 75, (1. Aufl., S.14-25). Münster: Waxmann.

Hug, T. (2011). Sondierungen im Spannungsfeld von Medienaktivismus und handlungsorientierter Medienpädagogik. Medienimpulse, 49(2), 1-19.

Kiesler, N. (2020). Medienkompetenzförderung im Lehramtsstudium der Goethe-Universität. Ein Projektbericht zur erfolgreichen Medienproduktion am Beispiel studentischer Erklärvideos. Zeitschrift MedienPädagogik, 17 (Jahrbuch Medienpädagogik), 477-506. https:// doi.org/10.21240/mpa-ed/jb17/2020.05.19.X.

Knezek, G., \& Christensen, R. (2016). Extending the will, skill, tool model of technology integration. Adding pedagogy as a new model construct. Journal of Computing in Higher Education, 28(3), 307-325. https://doi.org/10.1007/s12528-016-9120-2.

Kommer, S., \& Biermann, R. (2012). Der mediale Habitus von (angehenden) LehrerInnen. Medienbezogene Dispositionen und Medienhandeln von Lehramtsstudierenden. In R. Schulz-Zander, B. Eickelmann, H. Moser, H. Niesyto, \& P. Grell (Hrsg.), Jahrbuch Medienpädagogik, 9, 81-108. Wiesbaden: Springer VS.

Krumsvik, R. J. (2014). Teacher educators' digital competence. Scandinavian Journal of Educational Research, 58(3), 269-280. https://doi.org/10.1080/00313831.2012.726273.

Kultusministerkonferenz. (2016). Bildung in der digitalen Welt. Strategie der Kultusministerkonferenz. Berlin: Sekretariat der Kultusministerkonferenz. https://www.kmk.org/fil eadmin/Dateien/veroeffentlichungen_beschluesse/2018/Strategie_Bildung_in_der_dig italen_Welt_idF._vom_07.12.2017.pdf. Zugegriffen: 20. Mai. 2020.

Lemon, N., \& Garvis, S. (2016). Pre-service teacher self-efficacy in digital technology. Teachers and Teaching, 22(3), 387-408. https://doi.org/10.1080/13540602.2015.105 8594.

Levin, T., \& Wadmany, R. (2006). Teachers' beliefs and practices in technology-based classrooms. Journal of Research on Technology in Education, 39(2), 157-181. https://doi.org/ 10.1080/15391523.2006.10782478.

Mishra, P., \& Koehler, M. J. (2006). Technological pedagogical content knowledge. A framework for teacher knowledge. Teachers College Record, 108(6), 1017-1054. https://doi. org/10.1111/j.1467-9620.2006.00684.x.

Morin, A. J. S., Meyer, J. P., Creusier, J., \& Biétry, F. (2016). Multiple-group analysis of similarity in latent profile solutions. Organizational Research Methods, 19(2), 231-254. https://doi.org/10.1177/1094428115621148.

Muthén, L. K., \& Muthén, B. O. (1998-2016). Mplus 8.1 [Computer software]. Los Angeles: Muthén \& Muthén.

Ottenbreit-Leftwich, A. T., Glazewski, K. D., Newby, T. J., \& Ertmer, P. A. (2010). Teacher value beliefs associated with using technology. Addressing professional and student 
needs. Computers \& Education, 55(3), 1321-1335. https://doi.org/10.1016/j.compedu. 2010.06.002.

Rubach, C., \& Lazarides, R. (2019). Eine Skala zur Selbsteinschätzung digitaler Kompetenzen bei Lehramtsstudierenden. Entwicklung eines Instrumentes und die Validierung durch Konstrukte zur Mediennutzung und Werteüberzeugungen zur Nutzung digitaler Medien im Unterricht. Zeitschrift für Bildungsforschung, 2(78), 4. https://doi.org/10.1007/s35834019-00248-0.

Rubach, C., \& Lazarides, R. (2020). Digitale Kompetenzeinschätzungen von Lehramtsstudierenden fördern. Journal für lehrerInnenbildung, 20(1), 88-97. https://doi.org/10.35468/ jlb-01-2020_07.

Rubach, C., Lazarides, R., Brendel, N., \& Krauskopf, K. (2019). Learning by doing. Eine explorative Erhebung zur Förderung digitaler Kompetenzeinschätzungen durch die Verwendung digitaler Medien in der Hochschuldidaktik. In T. Ehmke, P. Kuhl, \& M. Pietsch (Hrsg.), Lehrer. Bildung. Gestalten. Beiträge zur empirischen Forschung in der Lehrerbildung (S. 164-175). Weinheim: Juventa Verlag.

Senkbeil, M., Schöber, C., \& Ihme, J. M. (2018). Fit fürs Studium? Computer- und informationsbezogene Basiskompetenzen Studierender und angehender Studierender. SchVw NRW, 7-8, 221-224.

Şimşek, Ö., \& Sarsar, F. (2019). Investigation of the self-efficacy of the teachers in technological pedagogical content knowledge and their use of information and communication technologies. World Journal of Education, 9(1), 196. https://doi.org/10.5430/wje.v9n $1 \mathrm{p} 196$.

Van Ackeren, I., Aufenanger, S., Eickelmann, B., Friedrich, S., Kammerl, R., Knopf, J., Mayrberger, K., Scheika, H., Scheiter, K., \& Schiefner-Rohs, M. (2019). Digitalisierung in der Lehrerbildung. Herausforderungen, Entwicklungsfelder und Förderung von Gesamtkonzepten. DDS-Die Deutsche Schule, 111(1), 103-119. https://doi.org/10.31244/dds.2019. 01.10 .

Open Access Dieses Kapitel wird unter der Creative Commons Namensnennung 4.0 International Lizenz (http://creativecommons.org/licenses/by/4.0/deed.de) veröffentlicht, welche die Nutzung, Vervielfältigung, Bearbeitung, Verbreitung und Wiedergabe in jeglichem Medium und Format erlaubt, sofern Sie den/die ursprünglichen Autor(en) und die Quelle ordnungsgemäß nennen, einen Link zur Creative Commons Lizenz beifügen und angeben, ob Änderungen vorgenommen wurden.

Die in diesem Kapitel enthaltenen Bilder und sonstiges Drittmaterial unterliegen ebenfalls der genannten Creative Commons Lizenz, sofern sich aus der Abbildungslegende nichts anderes ergibt. Sofern das betreffende Material nicht unter der genannten Creative Commons Lizenz steht und die betreffende Handlung nicht nach gesetzlichen Vorschriften erlaubt ist, ist für die oben aufgeführten Weiterverwendungen des Materials die Einwilligung des jeweiligen Rechteinhabers einzuholen.

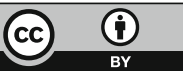

